\title{
A Synthetic DNA System for Programmed Pattern Formation and Sequence Construction
}

\author{
Ashok Nerella \\ M. Sc (Organic Chemistry), Lecturer in Chemistry, DR.BR. Ambedkar GMR Polytechnic for Women, Karimnagar
}

\begin{abstract}
In this paper, genetically engineered bacteria-based bio transceivers are investigated for transmission of information between bacteria populations, where bacteria can generate and respond to the molecular signals. A biochemical model of biological circuits is presented, and both analog and digital signaling are studied. The challenges in connecting basic biological circuits to build these blocks are revealed. Encryption performance is compared with our proposed work and the simulations are done with MATLAB simulation environment using physical layer functions where bacterial communication is modeled as transmitters and receivers.
\end{abstract}

Keywords: MATLAB, DNA System, Sequence Construction, Programmed Pattern Formation.

\section{INTRODUCTION}

Bacteria communicate with one another but, since they have neither mouth nor ears, they use chemical signals instead of sounds. They release small molecules into their environment and respond to the molecules of other bacteria. Such molecules are known as "auto-inducers". Based on their structure and concentration in the immediate environment, the bacteria are able to determine how many other bacteria are in the neighborhood. This type of chemical communication is called "quorum sensing". Auto-inducers typically regulate characteristic bacterial properties, which are much more effective when they are performed by a large number of cells, such as luminescence, the production of antibiotics, the formation of bio films, or the production of toxins.

Scientists in the group "Microbial Communication" investigate the mechanisms of quorum sensing in Streptococcus mutans, a human pathogen that causes caries, and in Dinoroseo bactershibae, a representative of the Roseobacter Group. They study the functions of genes and proteins using molecular methods that include gene knock-outs, micro-arrays, mRNA sequencing, and hetero logous expression of proteins. They have developed new test systems for finding inhibitors of quorum sensing. A very interesting source of such inhibitors is the Myxo bacteria, a group of soil bacteria that form fruiting bodies, which produce a large diversity of novel chemical structures with unknown biological functions. These quorum sensing inhibitors may eventually be used as novel anti-infectives. Such drugs are not required to kill the pathogen; rather they are intended to weaken its capability to make us sick.

Bacteria communicate and coordinate their activities through chemical signals that either diffuse through the extracellular environment or remain cell associated. As with the global surveillance of human chatter, research activity focused on microbial communication mechanisms has intensified over recent years. The study of bacterial communication systems is an extremely active area of microbiology and has generated a significant paradigm shift in the way we perceive and examine microbial populations.

\section{SIGNALING BASICS: LANGUAGES AND LOGIC}

Social and antisocial behavior in bacteria. Bacteria exudes a wide range of compounds into their external environment and also elaborate molecules that extend from their cell surfaces. Members of a growing list of diffusible molecules and cell-associated externalized structures function as signals (Table 1).

Some of the first recognized diffusible signaling mechanisms were described as "auto induction," reflecting the observation that the bacteria themselves were the source of the signal (70). Some bacterial signals may function as simple auto induction circuits, perhaps utilizing the stability of the signal as an indirect measure of relevant environmental parameters, such as $\mathrm{pH}$ or flow.

Most bacteria, however, are tuned to higher concentrations of the self-produced signals than that which individual cells can attain $(21,22)$. The term quorum sensing collectively describes these systems, in that inducing levels of signal require a minimum bacterial population density referred to as a quorum. Although quorum sensing $(84,88)$. In some cases, bacteria unable to synthesize their own signal are capable of responding to signals of other bacteria, eavesdropping on their competitors, or synchronizing with collaborators. There are also examples of interference, in which microbes and host organisms release signaling inhibitors or actively degrade bacterial signal molecules (107). Finally, there are multiple ways by which bacteria can communicate through cell contactdependent mechanisms in which signals elaborated on the cell surface interact to stimulate concerted behaviors. 


\section{REGULATORY NETWORKS: EXPANDING THE REACH OF COMMUNICATION}

Although the fundamental mechanisms for sensing and responding to signal molecules can be relatively simple, the potential complexity of the signaling networks they control is impressive. Many bacteria that inhabit complex environments experience multiple signals, some quite similar to their own, and thus they must discriminate between true signals and background noise. In addition, many bacteria recognize and respond to multiple chemically distinct signals. These signals may be integrated to amplify expression of a particular gene(s) or independently control transcription of discrete sets of genes; thus, the regulatory cascades involved in the response can be staggeringly complex.

\section{PHYSICAL INTERACTIONS OF BACTERIA}

Bacterial cell-cell signaling can occur through physical interaction with a neighboring cell or through the receipt of a diffusible signal molecule. In many cases, such as bio film formation or fruiting body formation, these interactions allow bacteria to function cooperatively and form complex structures.

Contact-dependent signaling in Myxo coccusxanthus. The soil bacterium M. Xanthus undergoes a complex developmental pathway, involving several different diffusible and cell contact-dependent signals, to form fruiting bodies and sporulate (46). Motility plays an important role in this process and is a target for signaling. Two motility mechanisms are simultaneously employed by M. Xanthus to move across surfaces; social (S) motility involves multiple cells that move via the action of type IV Pili, and adventurous (A) motility propels cells along surfaces by extrusion of extracellular slime through "nozzles" (45). The balance between these two distinct forms of motility, their regulation by environmental signals, and their role in M. Xanthus development are active areas of investigation. Trish Hartzell and Hera Vlamakis presented findings linking specific regulatory pathways, the MglARas-type GTPase and one of eight Che-type clusters from M. Xanthus, respectively, to the control of motility

M. Xanthus develops fruiting bodies from simpler cell aggregates, in part, through a series of cell reversals (46). When two cells meet end to end, they can exchange a contact-dependent cue called C-signal (Table 1) (49). This signal involves the Csg A protein processed to a $17-\mathrm{kDa}$ form, localized to the cell surface, and then recognized by presumptive C-signal receptors on colliding cells (59). Receipt of $\mathrm{C}$-signaling results in an increase in gene expression of the act $\mathrm{ABCDE}$ operation, which upregulates csgA expression, thus elevating $\mathrm{C}$-signal production. C-signal receipt also causes phosphorylation of the regulator FruA. This event influences the methylation/ demethylation state of the Che-like proteins FrzCD, which in turn affects phosphorylation of FrzE.
FrzE phosphorylation influences waves and streaming of aggregating M. Xanthus cells. Finally, FruA phosphorylation also impacts sporulation. Increased Csignaling is required for each of the three developmental events (waves, streaming, and sporulation) (47). Dale Kaiser presented new data on the stages of fruiting body formation (D. Kaiser, CCCB-04, abstr. S4:2). Kaiser described the end stages of fruiting body formation as a three-dimensional cell traffic jam, such as might occur at the Place de la Concorde if cars could climb on top of each other. Computer simulations of fruiting body formation based on C-signaling rules for each developmental stage accurately predict many aspects of fruiting body formation. These findings suggest that the signaling and motility mechanisms established to date are sufficient to account for much of fruiting body formation. Signaling and bio film formation. Bacteria that reside within bio films, surface-adherent communities, have extensive opportunities to communicate and physically interact. In some cases, communication is required to properly assemble the bio film $(12,40)$. In the gram-positive bacterium E. faecalis, oligo peptide-mediated signaling occurs through the Fsr AB two-component system (69). Work from two different groups demonstrated that the FsrA-controlled gelA gene, encoding a zinc metallo protease, is required for E. faecalis bio film formation (28, 52). In addition to its role in bio film formation, GelE functions in determining the length of the chains formed by E. faecalis cells, promoting degradation of mis folded surface proteins, and controlling the levels of the conjugation pheromone in culture supernatants (101). Marta Perego described the construction of strains with mutations in each of the 18 two-component systems of $\mathrm{E}$. faecalis (27; M. Perego and L. Hancock, CCCB-04, abstr. S4:3). Of these, only fsr was required for bio film formation, suggesting that other factors required for bio film formation might be controlled via alternate regulatory networks.

Bacteria in nature are often in mixed communities. It is clear that interactions among multiple bacterial taxa are quite complex, and even the presence of two bacterial species can significantly complicate matters. Ding ding An from Matt Parsek's lab described the results of competition experiments between $\mathrm{P}$. aeruginosa and A. tumefaciens in dispersed liquid culture and in bio films (D. An et al., CCCB-04, abstr. S7:6). In liquid culture, P. Aeruginosa cells numerically dominated A. tumefaciens cells, and this was dependent on the AHL synthases LasI and RhlI. In bio films formed on abiotic surfaces, P. Aeruginosa also numerically dominated $\mathrm{A}$. tumefaciens by developing into a thick blanket that covered the A. tumefaciens bio film. Blanketing by P. A eruginosa was diminished when either type IV pili (pilA) or flagella (flgK) were defective, although these mutants were unaffected for competition in liquid culture. The Las and Rhl signaling mutants were only modestly reduced in blanketing efficiency, suggesting that the competitive mechanisms of P. Aeruginosa were different in the two distinct environments. Supporting this 
idea, A. Tumefaciens more effectively competed with P. Aeruginosa during bio film formation on plant roots.

\section{HOST-MICROBE COMMUNICATION}

In addition to mediating chatter among different bacterial species, signals are clearly exchanged with or intercepted by eukaryotic hosts. Signals originally thought to be dedicated to inter bacterial communication can be recognized by eukaryotes, inducing specific responses. Zoospores of the marine alga Ulvaintestinalis respond to the presence of AHL-producing derivatives of Vibrio anguillarum and synthetic AHLs, enhancing their colonization of surfaces (44; D. Wheeler et al., CCCB-04, abstr. S6:4)

Plants also mount extensive responses to AHLs (64). Conversely, several different plants are known to produce quorum-sensing mimics (as yet uncharacterized), possibly to manipulate microbial rhizosphere populations (94). The marine alga Deli sea pulchra produces halogenated furan ones that disrupt AHL-based quorum sensing (23). Increasingly, it is apparent that metazoans have developed mechanisms by which they detect and in some cases manipulate microbial signaling pathways.

\section{BASIC DNA SEQUENCE}

DNA patterns are graphs of DNA or RNA sequences. Various functional structures such as promoters and genes, or larger structures like bacterial or viral genomes, can be analyzed using DNA patterns.

The technique was described in 2012 by Paul Gagniuc and Constantin Ionescu-Tirgoviste. They adapted algorithms from cryptography and optical character recognition to make their graphs. To graph a DNA pattern, two values, kappa index of coincidence and the total percentage of cytosine plus guanine $(\mathrm{C}+\mathrm{G}) \%$ are calculated from a sliding window which is "circulated" over the DNA sequence. The kappa index of coincidence measures the degree of organization or randomness of a sequence.

The analysis of such two-dimensional patterns can be performed by considering their shape and density (using optical character recognition algorithms) and the trend-line of the points. Inside a pattern, long homo polymeric tracts will be plotted in the upper part of the pattern (relative to the nucleotide frequency of the entire sequence) and tandem short tracts will be plotted in the middle of the pattern.

As the homo polymeric tracts become shorter and shorter (up to di- or tri- nucleotide formations), the kappa value decreases and the point on the pattern will be placed also in the middle, but lower on the Y-axis. All the values generated by the same repetitive sequences will be positioned in exactly the same point on the pattern (total points inside the pattern $=$ promoter length - sliding window length).

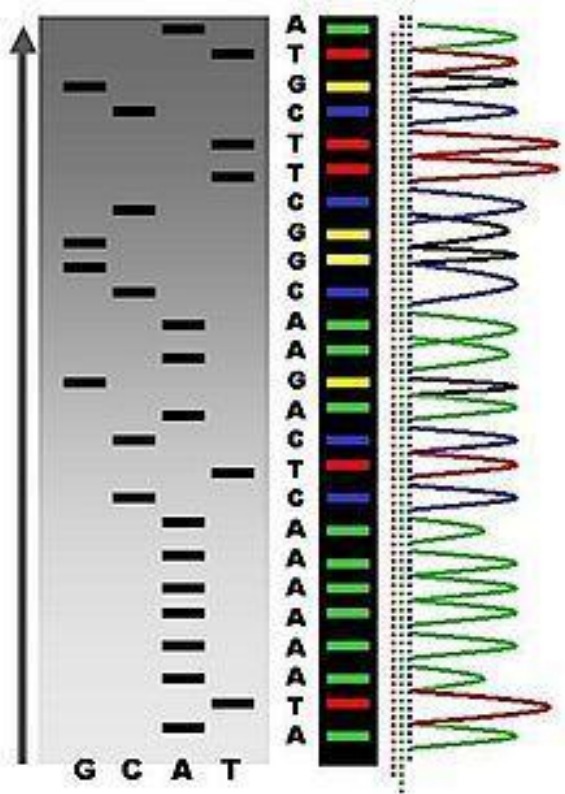

\section{CANONICAL STRUCTURE OF DNA}

The canonical structure of DNA has four bases: thymine $(\mathrm{T})$, adenine $(\mathrm{A})$, cytosine $(\mathrm{C})$, and guanine $(\mathrm{G})$. DNA sequencing is the determination of the physical order of these bases in a molecule of DNA. However, there are many other bases that may be present in a molecule. In some viruses (specifically, bacteriophage), cytosine may be replaced by hydroxy methyl or hydroxy methyl glucose cytosine. In mammalian DNA, variant bases with methyl groups or phosphor sulfate may be found. Depending on the sequencing technique, a particular modification may or may not be detected, e.g., the $5 \mathrm{mC}$ ( 5 methyl cytosine) common in humans may or may not be detected

DNA sequencing is the process of determining the precise order of nucleotides within a DNA molecule. It includes any method or technology that is used to determine the order of the four bases-adenine, guanine, cytosine, and thymine - in a strand of DNA. The advent of rapid DNA sequencing methods has greatly accelerated biological and medical research and discovery.

Knowledge of DNA sequences has become indispensable for basic biological research, and in numerous applied fields such as medical diagnosis, biotechnology, forensic biology, virology and biological systemetics. The rapid speed of sequencing attained with modern DNA sequencing technology has been instrumental in the sequencing of complete DNA sequences, or genomes of numerous types and species of life, including the human genome and other complete DNA sequences of many animal, plant, and microbial species. An example of the results of automated chain-termination DNA sequencing. The first DNA sequences were obtained in the early 1970s by academic researchers using laborious methods based on two-dimensional chromatography. Following the development of fluorescence-based sequencing methods with a DNA sequencer, DNA sequencing has become easier and orders of magnitude faster. 


\section{EXISTING SYSTEM}

A new study reveals that these bacteria are using their electrical network for signal transmission, just like in a telephone system. This discovery by researchers from the NIOZ Royal Netherlands Institute for Sea Research and the VrijeUniversiteis Brussel (VUB) reveals a whole new form of electrical communication by micro-organisms, and the discovery could potentially lead to entirely novel bioelectrical applications.

\section{a. Bacterial electricity and bio-electrical applications}

In 1858, the first electrical signals were sent through a telegraph cable across the Atlantic Ocean. As a result, the latest gossip from London travelled to New York in less than ten minutes, where it previously took ten days to deliver a message by ship. The newly discovered microbial interaction works in a very similar way: the algae at the sediment surface produce the oxygen signal (the Londoners), the cable bacteria convert and transmit the signal over long distances (the telegraph system), and the biology in the deep sediment rapidly knows about the latest developments at the surface (the New Yorkers). "This mechanism, whereby bacteria are conveying electrical signals over centimeter-scale distances, completely changes the way we think of how microbial communities interact and communicate, "adds team leader Meysman. Moreover, if the mechanism could be harnessed in an engineered way, this could lead to promising future technological developments. „Now we have a microbe from the seafloor that somehow has evolved the enzymatic machinery to perform electrical signaling. If we can find out how our electro genitic bacteria are achieving this, this clearly offers great opportunities for further research into novel bio-electrical materials and technology, ${ }^{\text {"e emphasizes }}$ Meysman. „Maybe within some years, solar panels or smart phones will harbor minuscule conducting wires of bacterial origin.

Bacteria communicate with one another in a similar way to nerve cells in the brain, scientists have discovered. Researchers conducted an experiment that showed how a colony of bacteria acted in a co-ordinated fashion when it reached a certain size. The microbes 'talk' by means of electrical signals which pass in and out of their bodies by gate-keeper 'ion channel' proteins. In the experiment, microbes at the outer edge of the 'bio film' colony consisting of hundreds of thousands of bugs had unrestricted access to nutrients while those at the centre were at risk of being starved. The mechanism the bacteria used was surprisingly similar to a process in the human brain known as 'cortical spreading depression' which is linked to migraines and epileptic seizures.

'This suggests that many drugs originally developed for epilepsy and migraines may also be effective in attacking bacterial bio films, which have become a growing health problem around the world because of their resistance to antibiotics.'

\section{ELECTRICAL EQUIVALENT SYSTEM}

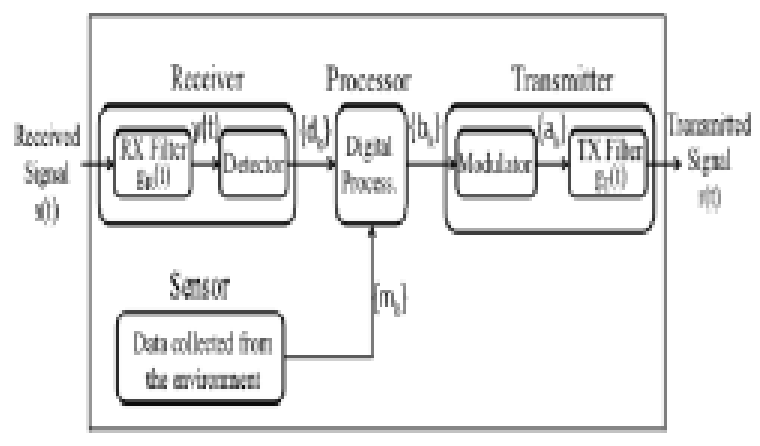

A transceiver for MC should be capable of sensing the environment, receiving signals from other MC devices, processing the received and sensed information and finally transmitting the processed information as molecular signals. Exploiting the mathematical abstraction we design representative biological circuits for each block of the bio transceiver architecture for QAM based base band modulation.

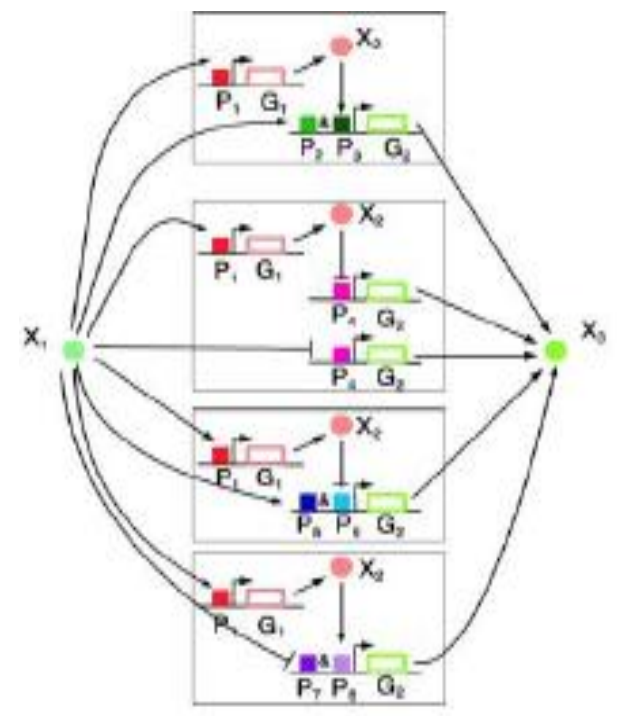

a. Transmitter

The function of the transmitter is the generation of molecular concentration signals $r(t)$ according to the data to be transmitted represented by the bit sequence fbkg. It consists of a modulator which creates symbols fakg from the bit sequence

\section{b. Receiver}

This block identifies the incoming molecular signal, measure its amplitude and convert the information encoded in the amplitude of the molecular signal to digital bits. For amplitude base modulation techniques, concentration band detector circuits can be used to identify different amplitude levels. The received signal $s(t)$ is first passed through a receive filter $\mathrm{gR}(\mathrm{t})$ and $\mathrm{y}(\mathrm{t})$ is obtained. Then, the detector determines the intended bits by threshold $\mathrm{y}(\mathrm{t})$. 


\section{BLOCK DIAGRAM}

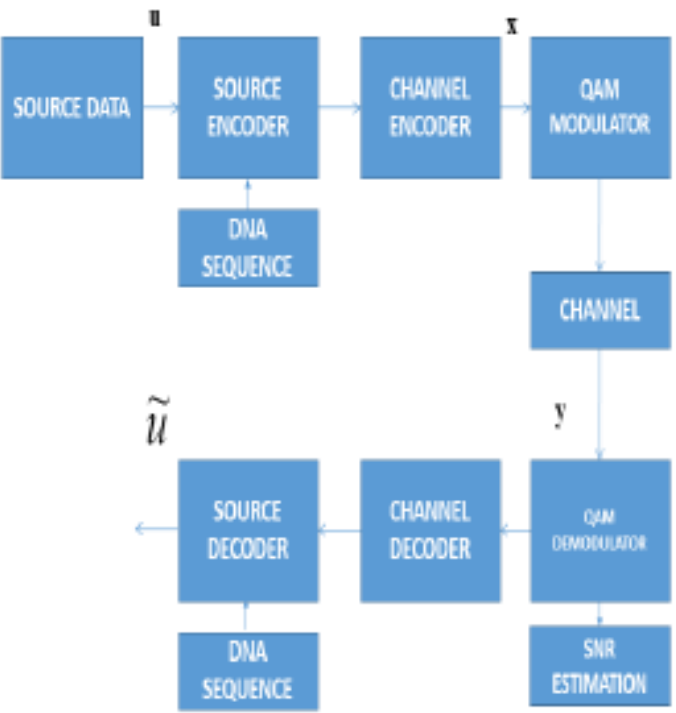

\section{CONCLUSION}

In this paper, we have implemented a bacterial movement, which struggles to obtain food. Such a motion is called as Brownian motion. While the bacteria interacts with each other, a chemical concentration is transferred to the other bacteria. We have calculated the Radial distribution of density of bacteria, and RMS distance of bacteria from food. With this work keeping as a base, we would extend this to a gated logic, encryption added communication system setup along with the detection of possible high density bacterial locations.

\section{REFERENCES}

[1] I. F. Akyildiz, J. M. Jornet, and M. Pierobon, "Nano networks: A new frontier in communications," Communication of the ACM, vol. 54, no. 11, pp.84-89, Nov. 2011.

[2] U. Alon, An introduction to systems biology: design principles of biological circuits, CRC press, 2006.

[3] P. E. M. Purnick, R. Weiss, "The second wave of synthetic biology: from modules to systems," Nat. Rev. Mol. Cell Bio., vol. 10, pp. 410-22, 2009.

[4] C. J. Myers, Engineering genetic circuits, CRC Press, 2011.

[5] H. H. McAdams, and A. Arkin, "Gene regulation: Towards a circuit engineering discipline," Curr. Biol., vol. 10, no. 8, pp. R318-R320, 2000 .

[6] T. Romeo, C. A. Vakulskas, and P. Babitzke, "Posttranscriptional regulation on a global scale: form and function of Csr/Rsm systems," Environmental Microbiology, vol. 15, no. 2, pp. 313-324, 2013.

[7] A. Tamsir, J. T. Jeffrey, and C. A. Voigt, "Robust multi cellular computing using genetically encoded NOR gates and chemical wires," Nature vol. 469, no. 7329, pp. 212-215, 2011.

[8] J. R.Van der Meer, "Bacterial sensors: synthetic design and application principles," Synth. Lect. on Synth.Biol., vol. 2, no. 1, pp. $4-5,2010$

[9] T. S. Gardner, C. R. Cantor, and J. J. Collins, "Construction of a genetic toggle switch in Escherichia coli," Nature, vol. 403, no. 6767, pp. 339-342, Jan. 2000.

[10] S. Basu, et al. "A synthetic multi cellular system for programmed pattern formation," Nature, vol. 434, no. 7037, pp. 1130-1134, Apr. 2005 . 